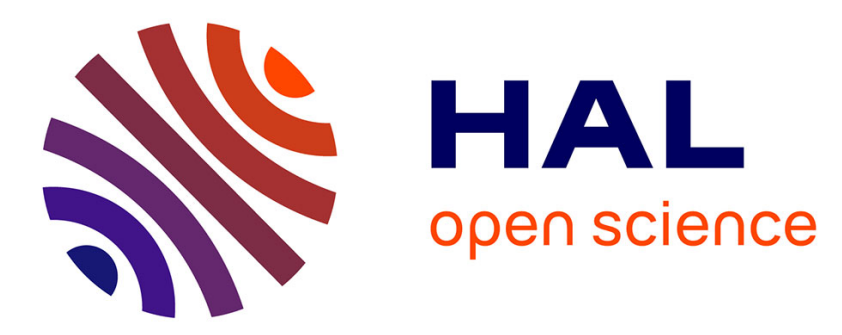

\title{
Large-bandwidth piezoelectric energy harvesting with frequency-tuning synchronized electric charge extraction
}

\author{
A. Brenes, Adrien Morel, D. Gibus, C.-S. Yoo, P. Gasnier, E. Lefeuvre, A.
} Badel

\section{- To cite this version:}

A. Brenes, Adrien Morel, D. Gibus, C.-S. Yoo, P. Gasnier, et al.. Large-bandwidth piezoelectric energy harvesting with frequency-tuning synchronized electric charge extraction. Sensors and Actuators A: Physical , 2020, 302, pp.111759. 10.1016/j.sna.2019.111759 . hal-03348327

\section{HAL Id: hal-03348327 \\ https://hal.science/hal-03348327}

Submitted on 18 Sep 2021

HAL is a multi-disciplinary open access archive for the deposit and dissemination of scientific research documents, whether they are published or not. The documents may come from teaching and research institutions in France or abroad, or from public or private research centers.
L'archive ouverte pluridisciplinaire HAL, est destinée au dépôt et à la diffusion de documents scientifiques de niveau recherche, publiés ou non, émanant des établissements d'enseignement et de recherche français ou étrangers, des laboratoires publics ou privés. 


\title{
Large-bandwidth piezoelectric energy harvesting with frequency-tuning synchronized electric charge extraction
}

\author{
$\underline{\text { A. Brenes }}^{1}$, A. Morel ${ }^{2,3}$, D. Gibus ${ }^{2,3}$, C.-S. Yoo ${ }^{4}$, P. Gasnier ${ }^{2}$, E. Lefeuvre ${ }^{5}$ and A. Badel ${ }^{3}$
}

\begin{abstract}
This paper reports, for the first time, experimental evidence of the effectiveness of the frequency-tuning synchronized electrical charge extraction technique (FTSECE) with a stronglycoupled generator. The ratio between the system bandwidth $(7 \mathrm{~Hz})$ and the natural bandwidth of the transducer $(0,688 \mathrm{~Hz})$ is $1017 \%$, surpassing previous demonstrations of synchronized charge extraction methods by a large extent. We prove that the bandwidth of the FTSECE system is only limited by the unideal characteristics of the circuit components and generator. This is a big advantage of FTSECE as opposed to other competitive methods where the bandwidth is intrinsically mathematically limited, even in the hypothetical presence of ideal components. We also propose a new circuit for the implementation of FTSECE, which allows the piezoelectric generator to be connected to the same ground as the control circuit. Our experimental setup based on the combination of a strongly-coupled piezoelectric generator and a FTSECE architecture allows operation at a maximum power plateau on a $+/-3.5 \%$ interval around the resonance frequency. The resulting full width at half maximum (FWHM) is $+/-10 \%$ around the resonance frequency, which corresponds to $+\mathbf{2 0 0 \%}$ compared to most up-to-date architectures designed for bandwidth enhancement, with the advantage of suppressing local minima in the power responses.
\end{abstract}

Keywords-Piezoelectric energy harvesting, resonant systems, synchronized switching, SECE, frequency tuning, FTSECE.

\section{INTRODUCTION}

$\mathrm{F}$ ROM an industrial perspective, the replacement of batteries by energy harvesters to power microsystems requires improvements in device size, weight, cost and performance. Harvesting wideband vibrations of the environment is hardly compatible with the frequency selectivity of high Q-factor resonators. Increasing the damping of the structure is the most obvious way to enlarge the system bandwidth but decreases the

This work was supported by the Korea Institute of Energy Technology Evaluation and Planning (KETEP) and the Ministry of Trade, Industry \& Energy (MOTIE) of the Republic of Korea (No. 20158510060040).

A. Brenes is with Institut Supérieur d'Electronique de Paris (ISEP), 28 rue Notre Dame des Champs, Paris, France (e-mail : alexis.brenes@isep.fr). A. Morel and D. Gibus are with Université Grenoble Alpes, CEA, LETI, MINATEC, F-38000 Grenoble, France and SYMME, Université Savoie Mont- maximum achievable extracted power. This simple statement has already justified researches on innovative resonator designs [1] and conditioning circuits [2] allowing bandwidth broadening of energy harvesters without reducing the maximal available power.

Following this trend, several solutions have been described in previous literature. Most of these solutions take advantage of full-bridge (FB) rectifiers at an intermediate stage [3]. Even if this architecture is limited by the voltage drop of the diodes [3], which has justified several works on alternative rectifiers [4] or rectifier-free circuits [5], full-bridge-based architectures remain a viable solution for energy harvesting [6].

Based on this statement, many works have focused on the optimization of energy harvesting architectures using FB rectifiers [7]. The charge transfer from an intermediate stage to a battery - or supercapacitor - is operated by a DC-DC converter. Several DC-DC converter topologies may be considered [2], each one requiring specific Maximum Power Point Tracking (MPPT) algorithms and circuitry. Amongst various control strategies, a first technique called "Synchronous Electrical Charge Extraction" (SECE) has initially been studied by Lefeuvre et al. [8]. However, this technique is known to exhibit relatively low performances (in terms of harvested power and bandwidth) for strongly-coupled piezoelectric resonators [8]. To improve the performance of classical SECE in terms of maximum power and/or bandwidth, recent works have proposed simple modifications to the original circuit [9] [10] [11] [12] [13]. Following this trend, the phase-shift SECE (PSSECE) technique has proven its ability to enlarge the bandwidth of strongly-coupled energy harvesters [11]. A similar approach has also been adopted to increase the offresonance performance of series and parallel-SSHI architectures [14] [15]. Ultimately, the so-called FTSECE

Blanc, F-74000 Annecy, France. C.-S. Yoo is with Electronic Convergence Materials \& Device Research Center, Korea Electronics Technology Institute, Republic of Korea. P. Gasnier is with Université Grenoble Alpes, CEA, LETI, MINATEC, F-38000 Grenoble, France. E. Lefeuvre is with Centre for Nanoscience and Nanotechnology, Univ. Paris Sud - CNRS, Université ParisSaclay, France. A. Badel is with SYMME, Université Savoie Mont-Blanc, F74000 Annecy, France. 
technique has been proposed [16] [2], in which both the instant and duration of charge extraction are tuned depending on the mechanical oscillation frequency. Numerical simulation results have proven that this technique significantly enlarges the bandwidth of SECE-based energy harvesters. Recent experiments on ASIC circuits have even demonstrated the feasibility of such modified SECE for fully-autonomous piezoelectric energy harvesters [17]. However, no full analytical study has yet determined the theoretical performances of FTSECE, and, no experimental validation has yet proven the full potential of FTSECE with a very strongly coupled piezoelectric resonator.

In this paper, we provide the full analytical developments proving that, as long as all resistive losses (in the piezoelectric material, switches and inductors) may be neglected, the theoretical bandwidth of FTSECE is infinite. We also analyze the impact of losses on the bandwidth of the system. The results are illustrated on a strongly-coupled resonator specifically designed to that purpose.

This paper is organized as follows. In section II, we present the main assumptions of our study. In section III, we provide the schematic of our circuit and the full performance analysis of the FTSECE technique. The results are compared to classical SECE [8], tunable SECE [10] and PSSECE [11] techniques. In section IV, we give experimental evidence of the efficiency of the FTSECE technique compared to the other techniques.

\section{MODEL OF INERTIAL PIEZOELECTRIC DEVICE}

Linear inertial piezoelectric energy harvesters are usually composed of a layer of piezoelectric transducer stuck on a mechanical resonator (e.g. a cantilever). In this study, we focus on the impact of nonlinear conditioning circuits on energy harvesters powered by a linear SDOF (single-degree-offreedom) piezoelectric resonator. Even though the assumption of linearity of the resonator is sometimes debatable [18], it remains a reasonable and common basis to compare the performance of electronic circuits [19] [20]. For such a resonator, the SDOF lumped model depicted in Figure 1 provides a reliable representation around one of its resonance frequencies [20]. It is composed of an inertial mass $M$ suspended by a spring of stiffness $K$. The natural frequency of the system is written $\omega_{0}=\sqrt{K / M}$. The damper $c$ models the mechanical losses of the system. External displacement $y$ (acceleration $\gamma$ ) results in a mechanical displacement of the resonator base. The vibration is translated into a (normalized) relative displacement $x$ of the inertial mass with respect to the base. The normalized variables and normalization factors are reported in Table I.
TABLE I

NORMALIZATION FACTORS

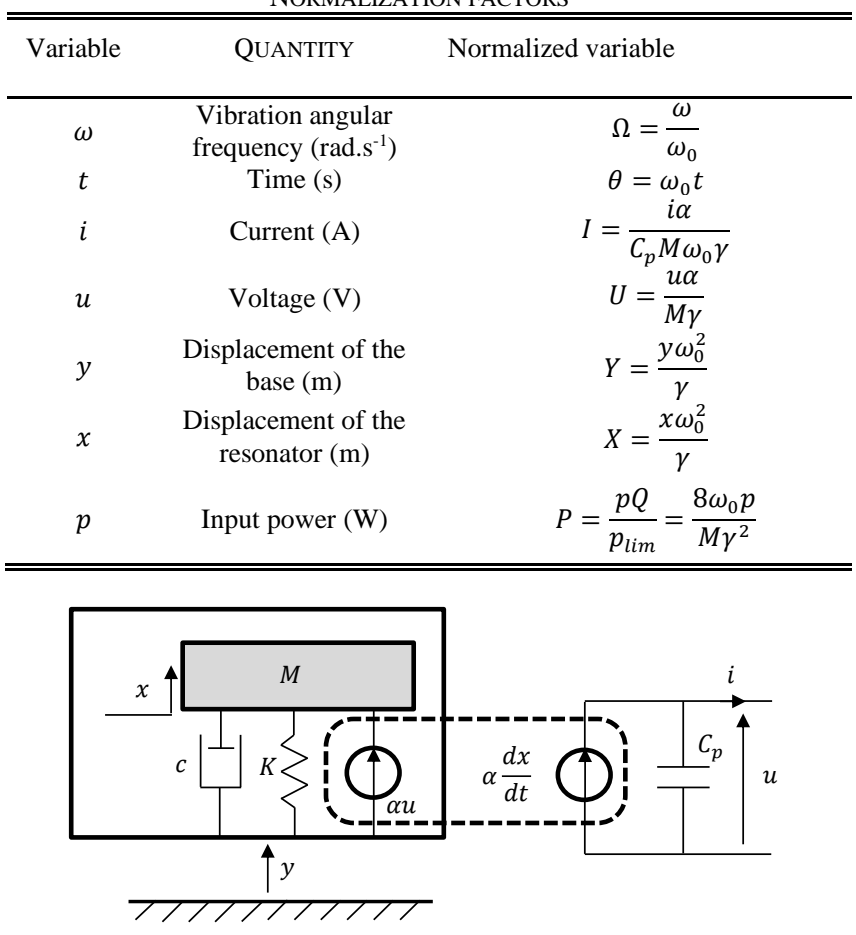

Figure 1: SDOF linear model of inertial piezoelectric energy harvesters.

The electromechanical coupling is achieved by the piezoelectric material through a piezoelectric coupling parameter $\alpha$. In this article, as in previous works, we choose to neglect the dielectric losses in the piezoelectric layer. This assumption has been validated on the resonators used for the experimental validation in section 4 [10]. Under this assumption, the governing equations of such a SDOF system are given by (.).

$$
\left\{\begin{array}{c}
M \frac{d^{2} x}{d t^{2}}+c \frac{d x}{d t}+K x+\alpha u=M \frac{d^{2} y}{d t^{2}} \\
i=\alpha \frac{d x}{d t}-C_{p} \frac{d u}{d t}
\end{array} .\right.
$$

Using the normalized variables given in Table I, (.) becomes (.)

$$
\left\{\begin{array}{c}
\frac{d^{2} X}{d \theta^{2}}+\frac{1}{Q} \frac{d X}{d \theta}+X+U=\frac{d^{2} Y}{d \theta^{2}} \\
I=k_{m}^{2} \frac{d X}{d \theta}-\frac{d U}{d \theta}
\end{array}\right.
$$

where $k_{m}^{2}=\alpha^{2} / K C_{p}$ is the expedient coupling coefficient and where $Q=M \omega_{0} / c$ is the mechanical quality factor.

Due to the electromechanical coupling, the amplitude and phase of the motion of the resonator are influenced by the piezoelectric voltage. The displacement of the resonator is assumed to remain sinusoidal despite the non-sinusoidal shape of $V$ (first-harmonic approximation). Hence, we write $X(\theta)=$ $X_{m} \sin \Omega \theta$, which is known to be a reasonable assumption for high-Q factor resonators [19], the higher harmonics being 
filtered out. For low Q-factor resonators, our analysis should be extended to account for higher harmonics.

To enlarge the bandwidth of piezoelectric energy harvesters, some authors focus on nonlinear mechanical resonators [21, 22]. Studying the combined effect of existing active control schemes with a nonlinear piezoelectric converter is the subject of very recent research and is out of the scope of this paper. A recent analysis of classical SECE combined with a bistable resonator can be found in Huguet et al. [23].

In the next section, we remind the principle of the FrequencyTuning SECE (FTSECE) technique [2], describe an electronic architecture for its implementation and analyze its optimal operating conditions.

\section{FREQUENCY-TUNING SECE (FTSECE)}

\section{A. Electronic architecture and power analysis}

The FTSECE technique [16] is obtained from a combination of the tunable SECE, first proposed by Richter et al. [9], and the PSSECE proposed by Lefeuvre et al. [11]. A first circuit for its implementation was proposed in [2] and experimentally demonstrated [24]. Here, we propose a new circuit (see Figure 2), which allows the piezoelectric generator to be connected to the same ground as the control circuit. This circuit includes a bidirectional switch $\mathrm{S}$ made of one NMOS and one PMOS transistor, whose control is synchronized with the piezoelectric voltage. Such a birectional switch has initially been proposed for vibration synchronized switch damping (SSD) in a different circuit [25]. Then, the principle of FTSECE lies in a twodegree-of-freedom optimization through the adjustment of instant and duration of the switch opening. When the switch is closed, during $t_{O N}$, an oscillation between the capacitance of the piezoelectric generator and the inductance $L$ is initiated at an angular frequency $\omega_{L C}=1 / \sqrt{L C_{p}}$. We assume that the frequency $\omega_{L C}$ is very high compared to the frequency $\omega$ of the acceleration and that the opening time $t_{O N}$ is inferior to half of the corresponding period $T_{L C}=\sqrt{L C_{p}}$. Under this assumption, closing the switch $S$ results in a quasi-instantaneous variation of $u$ (compared to the period of the mechanical oscillation). Contrary to another architecture proposed in previous literature [9] [10], the piezoelectric voltage $u$ may reach negative values (i.e. be inverted) when the switch $S$ is closed. The comparison between the piezoelectric voltage waveform obtained with classical SECE and FTSECE is illustrated in Figure 3, where $\varphi=\omega t=\Omega \theta$.

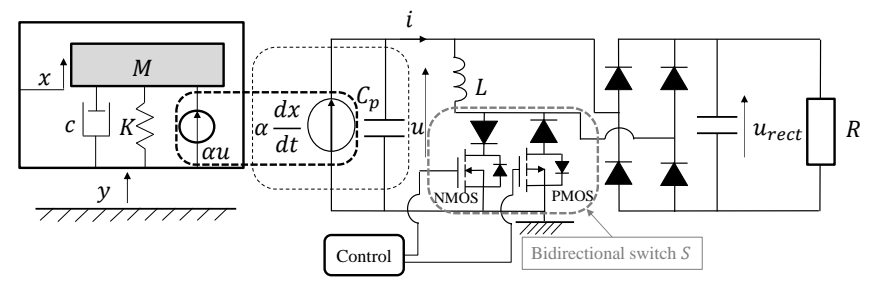

Figure 2: Interface circuit used for FTSECE.
Considering the tuning factor $\beta$ and the phase shift $\phi$ defined in Figure 3, the average power extracted from the piezoelectric transducer over each energy conversion cycle is given by (.).

$$
p=\frac{\omega}{2 \pi} C_{p} u_{m}^{2}\left(1-\beta^{2}\right) \cos ^{2} \phi .
$$

where $u_{m}$ is the peak piezoelectric voltage. As presented in Table I, we define the normalized power by $P=p Q / p_{\text {lim }}$, where $p_{\text {lim }}$ is the maximum average power which can be harvested by the piezoelectric energy harvester (.) [2] [26].

$$
p_{\text {lim }}=\frac{M^{2} \gamma^{2}}{8 c}=\frac{M \gamma^{2}}{8 \omega_{0}} Q
$$

This normalization leads to (.).

$$
P=\frac{4 \Omega}{\pi k_{m}^{2}} U_{m}^{2}\left(1-\beta^{2}\right) \cos ^{2} \phi .
$$

In this expression, the normalized peak voltage $U_{m}$ depends on the normalized frequency $\Omega$, but also on the tuning factor $\beta$ and the phase shift $\phi$. To determine the extracted power, one must first find the relationship between $U_{m}, \Omega, \beta$ and $\phi$.
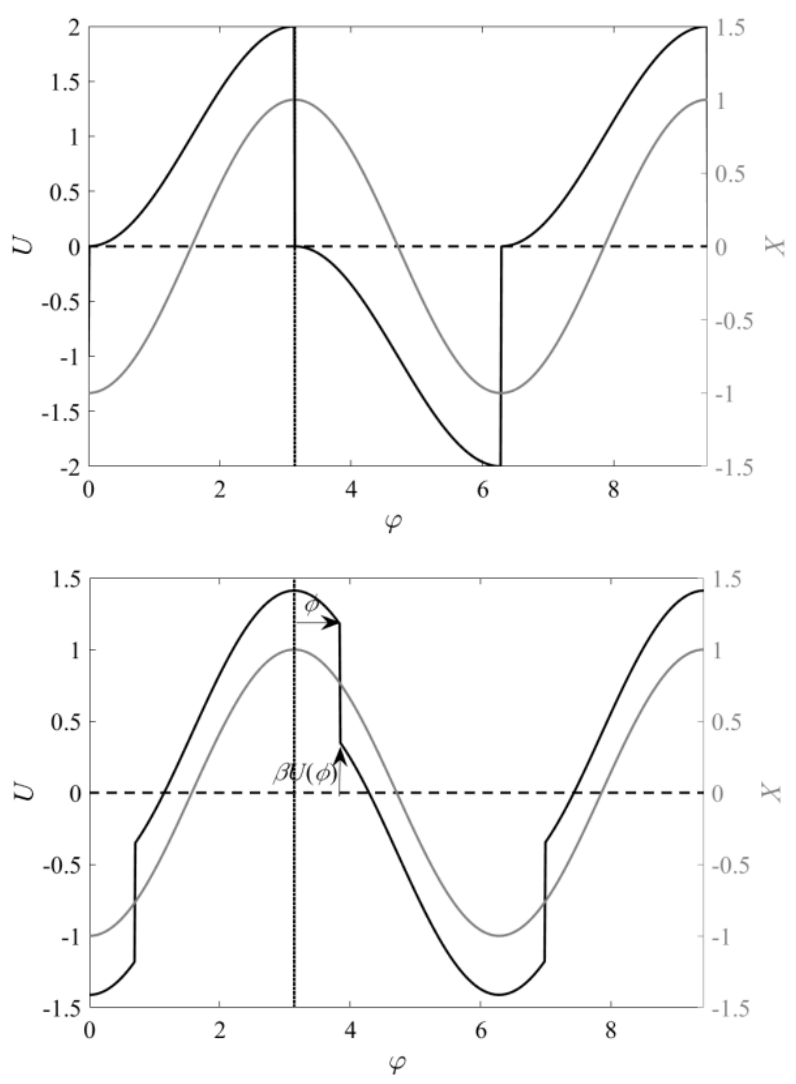

Figure 3: Typical waveforms in the SECE technique (up) and FTSECE technique (down).

To this purpose, the first harmonic approximation applied to (.) yields the relationship between the displacement of the resonator and the piezoelectric voltage. Under this assumption, only the fundamental component $U_{1}$ of voltage $U$ impacts the motion of the resonator when driven near its resonant 
frequency. According to the waveforms depicted in Figure 3, the normalized voltage $U$ is described by (.) where sign is the signum function (a.k.a. "sign function").

$$
\begin{aligned}
U(\varphi)=X_{m} k_{m}^{2}( & -\frac{1-\beta}{1+\beta} \cos \phi \operatorname{sign}[\sin (\phi-\varphi)] \\
& -\cos (\varphi))
\end{aligned}
$$

To determine the extracted power $p$, one still has to find out the normalized peak voltage $U_{m}$ (see (.)). From (.), the normalized peak voltage $U_{m}$ can also be expressed as a function of the magnitude $X_{m}$ of the relative displacement as (.).

$$
U_{m}=X_{m} \frac{2 k_{m}^{2}}{1+\beta}
$$

Then, the amplitude $X_{m}$ may be obtained from the method of harmonic balance [27]. This method requires the determination of the components $U_{p}$ and $U_{q}$ of the piezoelectric voltage $U$ which are respectively in-phase and in quadrature with respect to $X$. These terms are defined by (.).

$$
\left\{\begin{array}{c}
k_{m}^{2} X_{m} U_{p}=\frac{1}{\pi} \int_{0}^{2 \pi} U(\varphi) X(\varphi) d \varphi \\
k_{m}^{2} X_{m} U_{q}=\frac{1}{\pi} \int_{0}^{2 \pi} U(\varphi) \frac{d X}{d \varphi}(\varphi) d \varphi
\end{array}\right.
$$

From (.) and writing $Y(\Omega \theta)=\sin (\Omega \theta+\psi)$, the method of harmonic balance leads to (.).

$$
\left\{\begin{array}{c}
X_{m}\left(1-\Omega^{2}+k_{m}^{2} U_{p}\right)=\cos \psi \\
X_{m}\left(\frac{\Omega}{Q}+k_{m}^{2} U_{q}\right)=\sin \psi
\end{array}\right.
$$

where

$$
\left\{\begin{array}{c}
U_{p}=1+\frac{2}{\pi} \frac{1-\beta}{1+\beta} \sin 2 \phi \\
U_{q}=\frac{4}{\pi} \frac{1-\beta}{1+\beta} \cos ^{2} \phi
\end{array}\right.
$$

Finally, combining the two equations (.) leads to the expression of the amplitude $X_{m}$ of the mechanical oscillation, which depends on the acceleration frequency, the tuning factor $\beta$ and the phase shift $\phi$ through (.).

$$
\begin{aligned}
& X_{m}^{2} \\
& {\left[1-\Omega^{2}+k_{m}^{2}\left(1+\frac{2}{\pi} \frac{1-\beta}{1+\beta} \sin 2 \phi\right)\right]^{2}+\left[\frac{\Omega}{Q}+k_{m}^{2} \frac{4}{\pi} \frac{1-\beta}{1+\beta} \cos ^{2} \phi\right]^{2}}
\end{aligned}
$$

Finally, the expression (.) of the average power $P$ is derived from the combination of (.), (.) and (.). When $\beta=0$ and $\phi=0$, equation (.) is identical to the analytical expression of the harvested power calculated by Tang et al. for the SECE technique [28].

$$
=\frac{1-\beta}{\pi(1+\beta)} \frac{16 k_{m}^{2} \Omega \cos ^{2} \phi}{\left[1-\Omega^{2}+k_{m}^{2}\left(1+\frac{2}{\pi} \frac{1-\beta}{1+\beta} \sin 2 \phi\right)\right]^{2}+\left[\frac{\Omega}{Q}+k_{m}^{2} \frac{4}{\pi} \frac{1-\beta}{1+\beta} \cos ^{2} \phi\right]}
$$

To optimize the harvested power at a given frequency $\Omega, \beta$ and $\phi$ must be tuned properly. From (.), the optimal conditions (.) on $\beta_{\text {opt }}$ and $\phi_{\text {opt }}$ leading to the maximal harvested power can be obtained, for instance with a symbolic calculation software (Wolfram Mathematica in our case). These expressions of $\beta_{\text {opt }}$ and $\phi_{\text {opt }}$ lead to $P=Q$ at any frequency. A previous approximated expression had already been provided in [24] for sufficiently weak coupling $\left(k_{m}^{2} \ll 1\right)$ but (.) is the exact analytical result.

$$
\left\{\begin{array}{c}
\phi_{o p t}=-\arctan \left[\frac{Q\left(1+k_{m}^{2}-\Omega^{2}\right)}{\Omega}\right] \\
\beta_{o p t}=\frac{4 k_{m}^{2} Q \Omega-\pi\left[\Omega^{2}+Q^{2}\left(1+k_{m}^{2}-\Omega^{2}\right)^{2}\right]}{4 k_{m}^{2} Q \Omega+\pi\left[\Omega^{2}+Q^{2}\left(1+k_{m}^{2}-\Omega^{2}\right)^{2}\right]} \\
P(\Omega)=Q, \quad \forall \Omega
\end{array}\right.
$$

The specific case $\Omega=\sqrt{1+k_{m}^{2}}$ leads to $\phi_{\text {opt }}=0$, which highlights that the maximal value achieved by $\beta_{\text {opt }}$ at resonance is the same as the maximal value predicted in the case of tunable SECE [10]. In (.), one can see that the maximum normalized power $p=p_{\text {lim }}$ (i.e. $P=Q$ ) can theoretically be achieved at any frequency. This proves the ultimate effectiveness of the charge extraction operated by the FTSECE technique, where the theoretical bandwidth is infinite. To illustrate this statement, we report, in Figure 4, the evolution of $P$ for the classical SECE technique [8], tunable SECE [10], PSSECE [11] and the FTSECE technique. As already described in the literature, the SECE technique is unable to reach the maximum power $p=$ $p_{\text {lim }}$ (i.e. $P=Q$ ), except at the resonance frequency $\Omega=$ $\sqrt{1+k_{m}^{2}}$ and for one specific value of $k_{m}^{2} Q=\pi / 4$. In the other cases, the optimal values of the tunable parameters $\beta_{\text {opt }}$ and/or $\phi_{\text {opt }}$ are also given. On Figure 4-d (FTSECE), the three curves $P(\Omega)$, corresponding to three different values of $k_{m}^{2}$, are superimposed.

Two criteria can be used to compare the harvesting methods. The first criterion relates to the bandwidth of the system. With respect to this indicator, FTSECE largely outperforms the others in terms of bandwidth, which is theoretically unlimited. The second criterion relates to the peak harvested power. For resonators with low or medium electromechanical coupling (red and blue curves), FTSECE and tunable SECE are the only methods which achieve the maximum power $p_{\text {lim }}$ (i.e. $P=Q$ ). For weakly-coupled resonators $\left(k_{m}^{2} Q<\pi / 4\right)$, FTSECE does not bring any improvement to tunable SECE in terms of maximal harvested power (red curves) but only in terms of bandwidth. In practice, several experimental constraints actually limit the performance of the FTSECE technique. These limitations are explained in section B.

\section{B. Technical limitations of FTSECE \\ 1) Limitation due to inductor imperfections}

As one can notice in Figure 4, as the frequency gets farther from the natural frequency of the resonator, the optimal values of $\beta$ and $\phi$ tend to -1 and $\pm \pi / 2$ respectively. The condition $\beta=-1$ cannot be fullfilled in practice due to the intrinsic 


\section{$>$ REPLACE THIS LINE WITH YOUR PAPER IDENTIFICATION NUMBER (DOUBLE-CLICK HERE TO EDIT) <}

resistance of the inductance [29]. This resistance induces dissipation during the LC-oscillation when the switch is closed. In practice, $\beta$ is then bounded by a negative value $\beta_{\min }$ which verifies [30]:

$$
\beta_{\min } \approx-\exp \left[-\frac{\pi}{\sqrt{4 Q_{\text {ind }}^{2}-1}}\right]
$$

where $Q_{\text {ind }}$ is the inductor quality factor $\left(Q_{\text {ind }}=\frac{1}{R} \sqrt{\frac{L}{C_{p}}}\right)$. Hence, a limitation to the system performance relates to the practically-achievable lower bound $\beta_{\min }$ of $\beta$. The evolution of $\beta_{\min }(L)$ in the case of a piezoelectric resonator with $C_{p}=$ $100 \mathrm{nF}$ is reported in Figure 5 for commercially-available inductors (data from [31]). In order to optimize the performance of our proposed architecture, one should use a very small inductance value to ensure $\beta_{\min } \leq-0.95$. However, a small inductance value is not favorable in terms of actuation of parasitic modes and requires a more complex control system (see sections III.B.2) and III.B.3)).
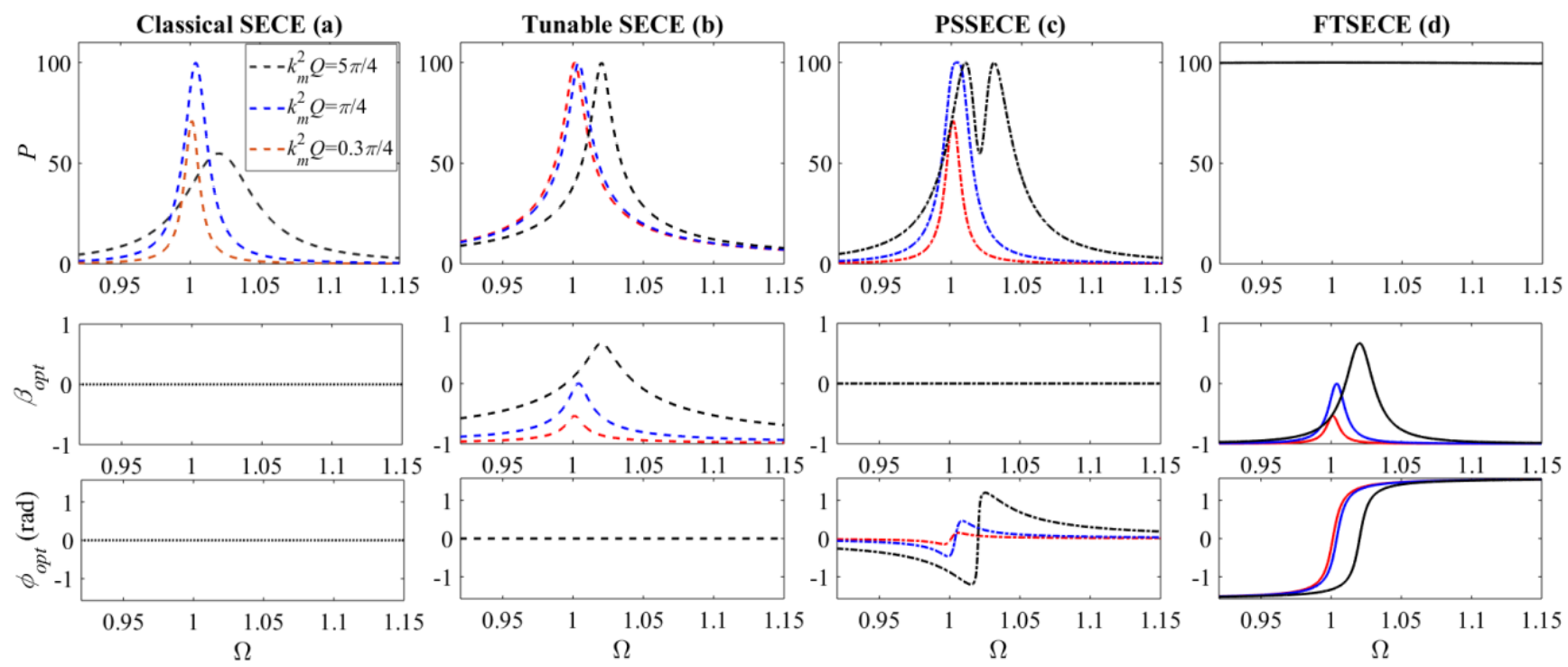

Figure 4: Normalized power vs. frequency and optimal values of $\beta$ and $\phi$ for SECE (a), tunable SECE (b), PSSECE (c) and FTSECE (d). Illustration given for $Q=100$ and three different values of $k_{m}^{2}$ (red: $k_{m}^{2} Q=0.3 \pi / 4$, blue: $k_{m}^{2} Q=\pi / 4$, black: $\left.k_{m}^{2} Q=5 \pi / 4\right)$. The power responses of FTSECE are all superimposed (blue, red and dark).

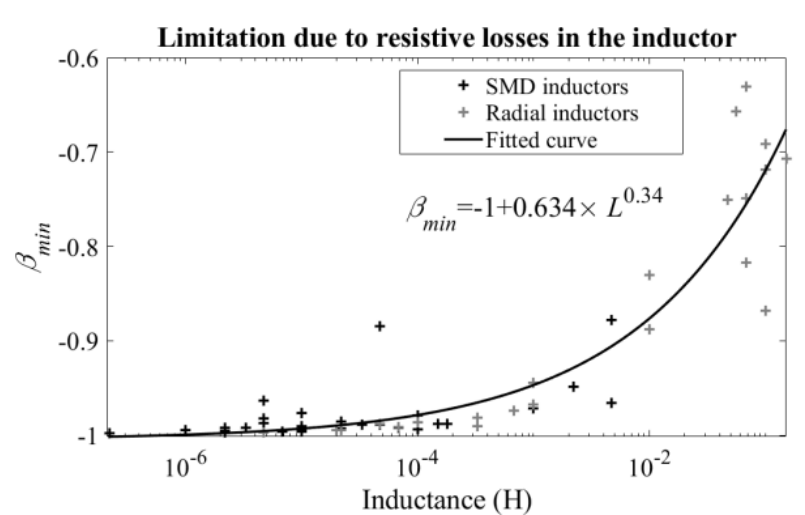

Figure 5: Lower bound of the tuning factor for commercially-available inductors (data from [31]). Example for a resonator with $C_{p}=100 \mathrm{nF}$.

The impact of the lower bound $\beta_{\min }$ on the bandwidth of the harvester is illustrated in Figure 6, for a FTSECE architecture limited to $\beta_{\text {min }}=-0.6$. Several conclusions can be drawn from the comparison between Figure $4-d$ and Figure 6 . As $\beta$ reaches its lower bound, the power $P$ starts to decrease. The limitation on $\beta$ leads to a different optimal phase shift $\phi_{\text {opt }}$. As soon as $\beta$ reaches $\beta_{\text {min }}$, the harvested power decreases and the optimal operating point verifies (.).

$$
\left\{\begin{array}{l}
\phi_{o p t}=-\arctan \left[\frac{4 k_{m}^{2}\left(1-\beta_{\min }\right) Q^{2}\left(1+k_{m}^{2}-\Omega^{2}\right)}{\pi\left(1+\beta_{\min }\right)\left[\left(\Omega^{2}+Q^{2}\left(1+k_{m}^{2}-\Omega^{2}\right)^{2}\right)\right]}\right] \\
P=\frac{16}{\pi} \frac{1-\beta_{\min }}{1+\beta_{\min }} \frac{k_{m}^{2} Q^{2} \Omega\left[\frac{\Omega^{2}}{Q_{m}^{2}}+\left(1+k_{m}^{2}-\Omega^{2}\right)^{2}\right]}{\left[\frac{\Omega^{2}}{Q^{2}}+\left(1+k_{m}^{2}-\Omega^{2}\right)^{2}+\frac{4}{\pi} \frac{1-\beta_{\min }}{1+\beta_{\min }} k_{m}^{2} \Omega\right]^{2}}
\end{array}\right.
$$

As one can see in Figure 6 and equation (.), in the case of FTSECE (as well as tunable SECE [9] or PSSECE [11]), all the curves (power, optimal $\beta$ and optimal $\phi$ ) are symmetrical with respect to $\Omega=\sqrt{1+k_{m}^{2}}$. 

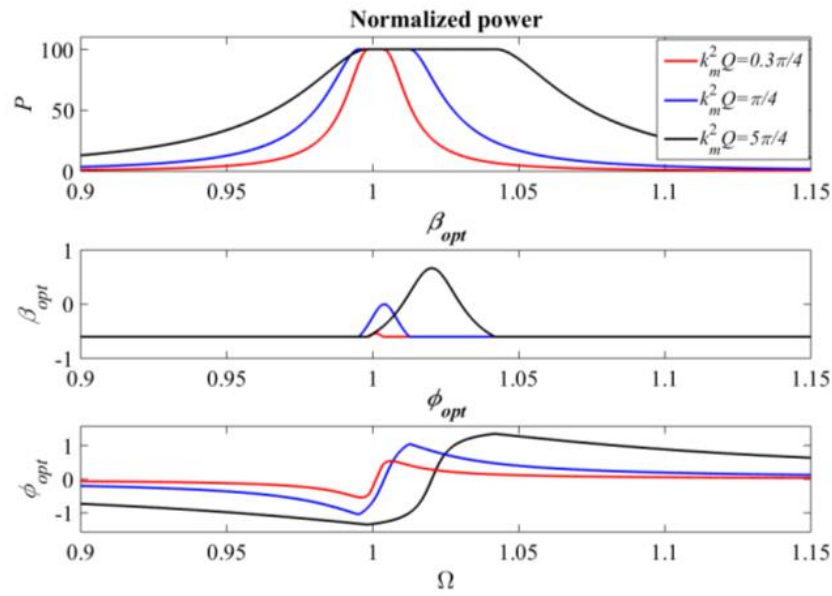

Figure 6: Normalized power vs. frequency for FTSECE limited to $\beta \geq$ -0.6 . Illustration given for $Q=100$ and three different values of $k_{m}^{2}$ (same as Figure 4).

Diminishing the lower bound $\beta_{\min }$ becomes a key issue, as the bandwidth is drastically modified by a small improvement of $\beta_{\text {min }}$. The constraint on $\beta_{\text {min }}$ can be alleviated using circuits including for instance multiple-step extraction as addressed by Lallart et al. [30].

\section{2) Limitation due to the complexity of the control scheme}

We have seen in III.B.1) that the best inversion tends to be obtained with small inductance values. However, the problem then comes at the control scheme level. If the inductor is very small, the inversion time becomes very short and it becomes complex for the control scheme to react and eventually stop the inversion before the end of the inversion, to tune $\beta$ precisely.

\section{3) Limitation due to higher resonance modes}

As written in section III.B.1), the performance of FTSECE is limited by the achievable lower bound $\beta_{\text {lim }}$ of $\beta$. To overcome this limitation, one should opt for a low-resistance and lowinductance inductor which yields to $\beta_{\min }$ close to -1 . However, such an inductor produces very sharp voltage drops which increase the risk of actuating higher resonance modes of the resonator. A balance should then be struck between, on the one hand, the minimization of $\beta_{\min }$ and, on the other hand, the energy losses into higher modes and the feasibility of the control scheme. If one wants $N$ steps of $\beta$, the response time of the electronic control must be below $\pi \sqrt{L C_{p}} / 2 N$. Other methods to limit this problem would be to perform the inversion in several steps.

4) Remarks on the limitation due to the dielectric losses in the piezoelectric layer

In addition to the limitations already discussed, another limitation relates to the dielectric losses in the piezoelectric layer. These losses would not only dissipate a portion of the power but also modify the displacement of the resonator and the piezoelectric voltage through the electromechanical coupling. Taking them into account, one can expect the real bandwidth to be narrower than predicted by our model. Indeed, as $\beta$ gets closer to -1 , the peak piezoelectric voltage drastically increases, resulting in a larger amount of power dissipated via dielectric losses in the piezoelectric layer. This becomes even more problematic as the dielectric losses depend nonlinearly on the electric field [32].

\section{5) Remarks about power consumption of active control system}

To discuss about the feasibility of FTSECE in a fullyautonomous system, the power consumption of the active control scheme is a significant issue. Previous studies have demonstrated the feasibility of active control schemes with integrated circuits $[17,33,34,35]$. These works indicate power consumptions between $0.3 \mu \mathrm{W}$ and a few microwatts (typically $3 \mu \mathrm{W})$. Based on this statement, the implementation of FTSECE makes no sense for applications where only a few microwatts are available and requires at least $10 \mu \mathrm{W}$ of harvested power.

\section{EXPERIMENTAL VALIDATION}

\section{A. Device structure and experimental setup}

The device used for the validation of FTSECE is specifically designed to maximize the piezoelectric coupling and minimize the chances of actuating parasitic mechanical modes (see Figure 7 ). To that purpose, two patches of high coupling PMN-PT X2B $(45 \times 10 \times 0.5 \mathrm{~mm})[36]$ have been bonded to a cantilever beam made of steel whose thickness is equal to $0.5 \mathrm{~mm}$. To ensure that the resonator remains single-mode, a thick mass of steel $(45 \times 10 \times 5 \mathrm{~mm})$ has been stuck to the sheet, close to the free end. The mass length has been chosen in order to homogenize the strain distribution in the piezoelectric material. The physical characteristics of the generator are summed up in Table II. More details about the design of the generator can be found in a paper dedicated to the mechanical aspects [37].

The resonator is clamped to a shaker (2075E-HT, The Modal Shop(C) driven by a dedicated power amplifier (2050E09, The Modal Shop(C). The speed and relative displacement of the mass of the resonator with respect to the base are measured thanks to a differential vibrometer (OFV 552 with OFV 5000 controller, Polytec(C). The acceleration of the base is measured using an accelerometer (M352C68, PCB (C) and set at an amplitude of $\gamma=0.4 \mathrm{~m} . \mathrm{s}^{-2}$. The inductor placed in the circuit is $L=1 H$, chosen for its low intrinsic losses (inductor quality factor $Q_{\text {ind }}=3.4$ ) so that $\beta \simeq 0.62$. In our setup, the necessity of such a large inductor comes from the sample time of the control scheme implemented in a dSPACEC RTI1103 real time interface, which cannot be lower than $5 \mu \mathrm{s}$. The future use of integrated electronic control will allow lower response times and thus smaller inductors.

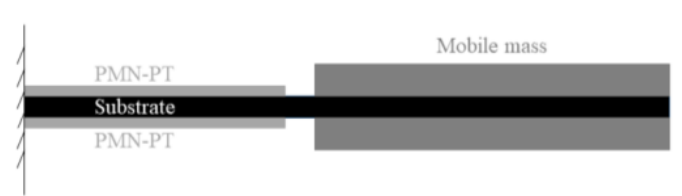




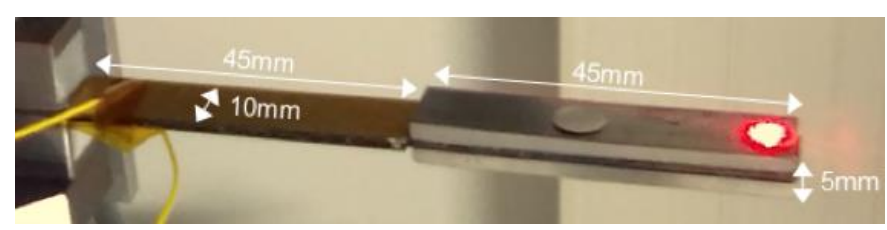

Figure 7: Cross-sectional view and picture of the strongly coupled piezoelectric generator.

The experimental setup is automated thanks to the dSPACE real-time control and acquisition board (Figure 8). The voltage driving the electromagnetic shaker is directly delivered from this control board, and its amplitude is regulated in order to maintain the shaker acceleration amplitude constant. The piezoelectric harvester electrodes are connected to the electronic circuit realizing the FTSECE strategy.

The measurements are performed at 50 vibration frequencies ranging from $24 \mathrm{~Hz}$ to $45 \mathrm{~Hz}$, and for a set of $30 \times 30$ values of tuning parameters $(\beta, \phi) \in[-0.7,1] \times\left[-90^{\circ}, 90^{\circ}\right]$. For each combination of tuning parameters $(\beta, \phi)$ and for each frequency, the voltages, mechanical displacement, speed and acceleration waveforms are recorded and stored in a computer

TABLE II

PIEZOELECTRIC GENERATOR CHARACTERISTICS

\begin{tabular}{ccc}
\multicolumn{3}{c}{ PIEZOELECTRIC GENERATOR CHARACTERISTICS } \\
\hline \hline \multirow{2}{*}{ Variable } & Quantity & Value \\
& Piezoelectric generator capacitance & $C_{p}=50 \mathrm{nF}$ \\
$C_{p}$ & Quality factor & $Q=80$ \\
$Q$ & Short-circuit resonance frequency & $f_{0}=27.51 \mathrm{~Hz}$ \\
$f_{0}$ & Expedient coupling coefficient & $k_{m}^{2}=0.156$ \\
$k_{m}^{2}$ & & \\
\hline \hline
\end{tabular}

thanks to the dSPACE acquisition board.

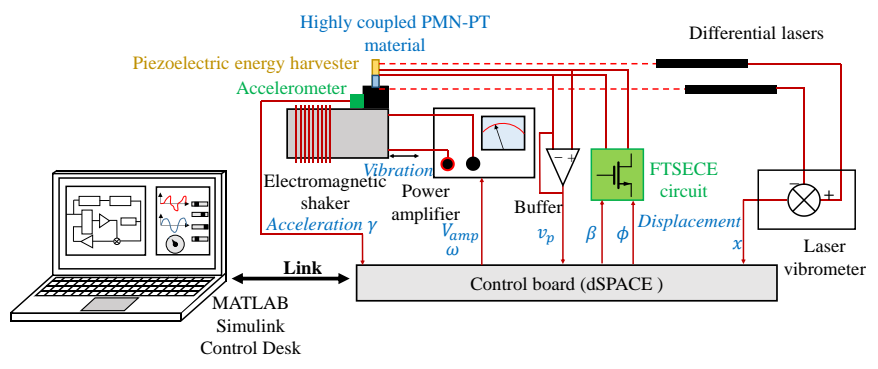

Figure 8: Experimental setup

\section{B. Experimental results}

The results obtained with FTSECE are reported in Figure 9. The theoretical response is based on the resonator characteristics given in Table II. The capacitance is measured with an impedance analyzer and the other parameters are estimated based on the power response of Fig. 9. One can notice a good agreement between our theory and the experiments. The remaining differences are assumed to come from higher resonance modes (see section 3.2.2) and the intrinsic dielectric losses in the piezoelectric layer (see section III.B.4)), which our model does not take into account. Based on the difference between the modeled and the experimental input power obtained, we estimate the energy losses in higher resonance modes to be lower than $10 \%$ of the available power when the
TABLE III NVPD FIGURE-OF-MERIT

\begin{tabular}{ccccccc}
\hline \hline Ref. & $\begin{array}{c}V \\
\left(\mathrm{~cm}^{3}\right)\end{array}$ & $\gamma(\mathrm{g})$ & $\begin{array}{c}f_{0} \\
(\mathrm{~Hz})\end{array}$ & $\begin{array}{c}p_{\max } \\
(\mathrm{mW})\end{array}$ & $\begin{array}{c}\frac{\Delta f}{f_{0}} \\
(\%)\end{array}$ & $\begin{array}{c}\mathrm{NPD}(\mu \mathrm{W} . \\
\left.\mathrm{mm}^{-3} \cdot \mathrm{g}^{-2}\right)\end{array}$ \\
\hline$[32]$ & 535 & 0.255 & 120 & 0.375 & $\mathrm{~N} / \mathrm{A}^{*}$ & 10.8 \\
{$[33]$} & 6750 & 0.25 & 100 & 4 & 7.1 & 9.5 \\
{$[34][35]$} & 464 & 0.2 & 76 & 0.139 & 5.2 & 0.75 \\
{$[36][37]$} & 9000 & 0.1 & 50 & 0.18 & 8 & 2.0 \\
{$[38]$} & 1400 & 0.41 & 116 & 1.4 & 4.1 & 5.95 \\
{$[38]$} & 1400 & 1.8 & 93.5 & 21.3 & 2.4 & 4.7 \\
This & 6300 & 0.041 & 27.5 & 0.093 & 12.7 & 8.8 \\
work & & & & & & \\
\hline \hline
\end{tabular}

*N/A: Not available

first bending mode is close to resonance. This proportion is relatively low due to the specific design of our generator, for which the frequency of the second mode is more than 10 times higher than the frequency of the first bending mode (obtained with FEM simulations). As expected, the power obtained with FTSECE exhibits a plateau (at $93 \mu \mathrm{W}$ ) with no local minimum. To compare this harvester with other ones from the literature, we use a common figure of merit (FoM) called "normalized power density" (NPD) defined by (.), where $V$ is the active volume of the device and $p_{\max }$ the maximum input power [38] [39] (one should remind that $p_{\max }=p_{\text {lim }}$ corresponds to the best-case scenario).

$$
\mathrm{NPD}=\frac{p_{\max }}{\gamma^{2} V}
$$

We obtain a NPD of $8.8 \mu \mathrm{W} \cdot \mathrm{mm}^{-3} \cdot \mathrm{g}^{-2}$, which is among the best state-of-the-art piezoelectric transducers with millimetric or centimetric dimensions (see examples in Table III), with a much larger relative bandwidth (see examples in Table III). The full width at half maximum of our system is $\Delta f= \pm 3.5 \mathrm{~Hz}$ corresponding to a normalized bandwidth $\Delta f / f_{0} \simeq 12.7 \%$.

To demonstrate the interest of FTSECE compared to other existing methods, we also plot the results obtained for classical SECE, tunable SECE and PSSECE in Figure 10. The experiments validate the most important conclusions captured by the model: the large bandwidth of our system with FTSECE compared to the other techniques and the improvement in terms of harvested power (more than 4 times compared to SECE).

We also report, in this figure, the output power (grey lines) collected in the storage capacitor at the end of the electrical chain. Even though the improvement of the electrical power efficiency of the SECE circuits remains a hot topic, higher peak power and larger frequency bandwidth are obtained at the output of our circuit. The main losses between input and output power take place in the inductor and in the piezoelectric layer, as discussed in sections III.B.1) and III.B.4), but also in the diodes (conduction losses) since the voltage amplitudes are typically between $1 \mathrm{~V}$ and $10 \mathrm{~V}$ (depending on the frequency). The resulting full width at half maximum is doubled compared to PSSECE, with no local minimum, which is a very significant improvement. 

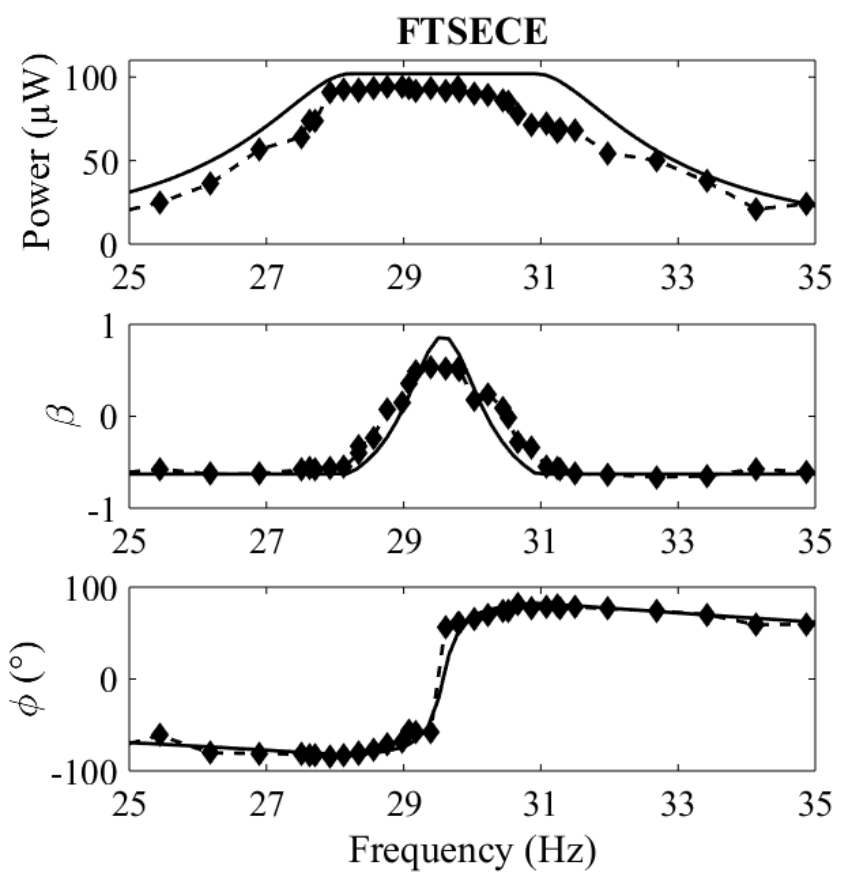

Figure 9: Experimental results for FTSECE (input power and control parameters). Measurements (diamonds) and theory (solid line).

\section{CONCLUSION}

In this paper, we have experimentally demonstrated wideband piezoelectric energy harvesting with a strongly-coupled piezoelectric energy harvester $\left(k_{m}^{2}=15.6 \%\right)$. We have proven that the resulting system bandwidth may be doubled compared to the most up-to-date bandwidth enhancing techniques. With the proposed resonator and architecture, we obtain more than $50 \mu \mathrm{W}$ input power on a $7 \mathrm{~Hz}$ bandwidth around $29 \mathrm{~Hz}$, for an acceleration amplitude of $0.041 \mathrm{~g}$, corresponding to a RMS acceleration of $0.028 \mathrm{~g}$. The normalized power density of our harvester is $8.8 \mu \mathrm{W} \cdot \mathrm{mm}^{-3} \cdot \mathrm{g}^{-2}$ and its normalized bandwidth is $12.7 \%$ of the short-circuit resonance frequency, which places it among the best piezoelectric transducers with millimetric or centimetric dimensions. In addition, we have shown that the use of FTSECE suppresses the local minimum exhibited by the power vs. frequency response caused by other state-of-the-art control schemes.

An automated procedure has been implemented, proving the performance of the FTSECE approach. A further theoretical analysis has allowed us to derive simple expressions of the optimal operating point of FTSECE. This theoretical analysis can be used as a basis for the optimization of the maximum power point tracking procedure. One remaining limitation to the use of FTSECE lies in the impact of the quality factor of the inductor under use for charge extraction. A high electrical quality factor is obtained for small inductor values, which implies that the control circuit response time must be short enough to provide accurate tuning of the charge extraction duration. This constraint can be alleviated using circuits including for instance multiple-step extraction. Design of a strongly-coupled piezoelectric resonator with a large intrinsic capacitance is another way to address this issue. These improvements are the subject of ongoing research.
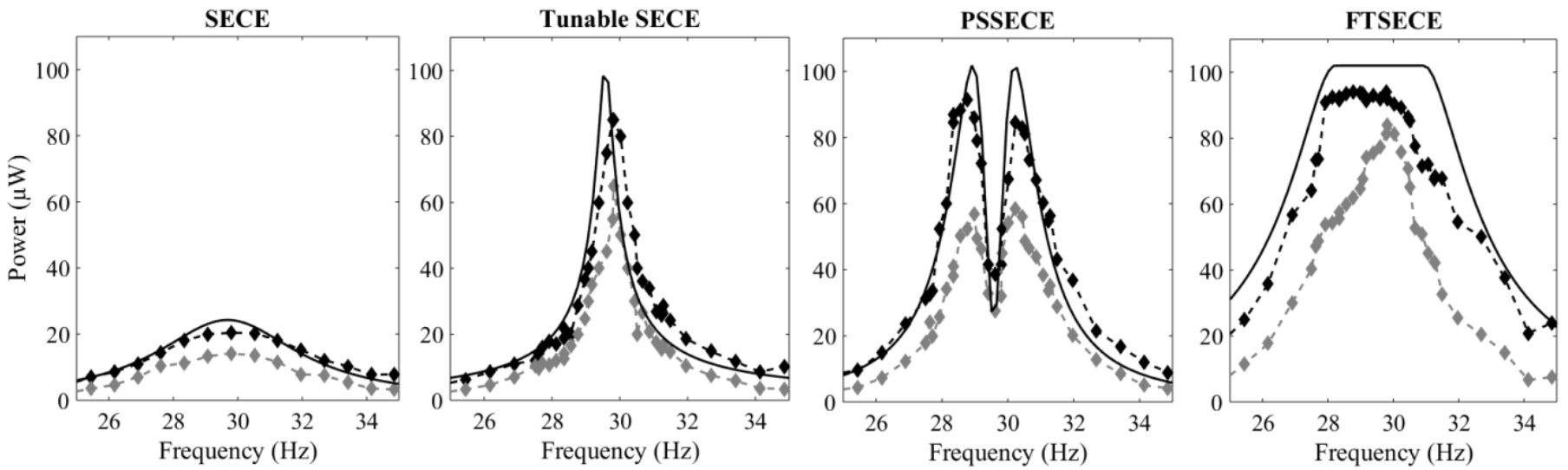

Figure 10: Experimental comparison: From left to right : Input power obtained with a matched resistive load, SECE, tunable SECE, PSSECE and FTSECE (dark diamonds). Output power (grey diamonds). For resistive matching, grey and black are superimposed. Theoretical response based on the resonator and inductor characteristics (solid dark line).

\section{REFERENCES}

[1] M. Ferrari, V. Ferrari, M. Guizzetti, D. Marioli and A. Taroni, "Piezoelectric multifrequency energy converter for power harvesting in autonomous microsystems," Sensors and Actuators A, vol. 142, pp. 329335, 2008.

[2] A. Badel and E. Lefeuvre, "Nonlinear Conditioning Circuits for Piezoelectric Energy Harvesters," in Nonlinearity in Energy Harvesting
Systems, E. Blokhina, A. E. Aroudi, E. Alarcon and D. Galayko, Eds., Cham, Springer, 2016, pp. 353-357.

[3] H. S. Kim, J.-H. Kim and J. Kim, "A Review of Piezoelectric Energy Harvesting Based on Vibration," International Journal of Precision Engineering and Manufacturing, vol. 12, no. 6, pp. 1129-1141, December 2011.

[4] Y. K. Ramadass and A. P. Chandrakasan, "An Efficient Piezoelectric Energy Harvesting Interface Circuit Using a Bias-Flip Rectifier and 
Shared Inductor," IEEE Journal of Solid-State Circuits, vol. 45, no. 1, pp. 189-204, January 2010.

[5] D. Kwon and G. A. Rincon-Mora, "A rectifier-free piezoelectric energy harvester circuit," in Circuits and Systems, 2009. ISCAS 2009. IEEE International Symposium on, Taipei, 2009.

[6] C. Peters, J. Handwerker and D. Maurath, "A Sub-500 mV Highly Efficient Active Rectifier for Energy Harvesting Applications," IEEE Transactions on Circuits and Systems I: Regular Papers, vol. 58, no. 7, pp. 1542-1550, June 2011.

[7] L. Tang, L. Zhao, Y. Yang and E. Lefeuvre, "Equivalent Circuit Representation and Analysis of Galloping-Based Wind Energy Harvesting," IEEE/ASME Transactions on Mechatronics, vol. 20, no. 2, pp. $834-844,2015$.

[8] E. Lefeuvre, A. Badel, C. Richard and D. Guyomar, "Piezoelectric energy harvesting device optimization by synchronous electric charge extraction," J. of Intelligent Material Systems and Structures, vol. 16, pp. $865-876,2005$

[9] A. Richter, A. Strobel, N. Joram, F. Ellinger, L. Göpfert and R. Marg, "Tunable Interface for Piezoelectric Energy Harvesting," in Systems, Signals \& Devices (SSD), 2014 11th International Multi-Conference on, Barcelona, 2014

[10] E. Lefeuvre, A. Badel, A. Brenes, S. Seok, M. Woytasik and C.-S. Yoo, "Analysis of piezoelectric energy harvesting system with tunable SECE interface," Smart Materials and Structures, vol. 26, no. 3, February 2017.

[11] E. Lefeuvre, A. Badel, A. Brenes, S. Seok and C.-S. Yoo, "Power and frequency bandwidth improvement of piezoelectric energy harvesting devices using phase-shifted SECE interface circuit," J. of Intelligent Material Systems and Structures, 2017.

[12] A. Morel, A. Badel, Y. Wanderoild and G. Pillonnet, "A unified N-SECE strategy for highly coupled piezoelectric energy scavengers," Smart Materials and Structures, vol. 27, p. 084002, 2018.

[13] A.Morel, G. Pillonnet, P. Gasnier, E. Lefeuvre and A. Badel, “" « Frequency tuning of piezoelectric energy harvesters thanks to a shortcircuit synchronous electric charge extraction," Smart Materials and Structures, 2018.

[14] P.-H. Hsieh, C.-H. Chen and H.-C. Chen, "Improving the Scavenged Power of Nonlinear Piezoelectric Energy Harvesting Interface at OffResonance by Introducing Switching Delay," IEEE Transactions on Power Electronics, vol. 30, pp. 3142-3155, 2015.

[15] L. Zhao, L. Tang, J. Liang and Y. Yang, "Synergy of Wind Energy Harvesting and Synchronized Switch Harvesting Interface Circuit," IEEE/ASME Transactions of Mechatronics, vol. 22, no. 2, pp. 10931103, 2017.

[16] A. Badel and E. Lefeuvre, "Wideband piezoelectric energy harvester tuned through its electronic interface circuit," Journal of Physics Conference Series, vol. 557, no. 1, 2014.

[17] Y. Cai and Y. Manoli, “A Piezoelectric Energy-Harvesting Interface Circuit with Fully Autonomous Conjugate Impedance Matching, 156\% Extended Bandwidth, and $0.38 \mu \mathrm{W}$ Power Consumption," in International Solid - State Circuits Conference (ISSCC), San Francisco, 2018.

[18] D. A. Hall, "Review - Nonlinearity in piezoelectric ceramics," Journal of Material Science, vol. 36, no. 19, p. 4575-4601, October 2001.

[19] Y. Liao and H. A. Sodano, "Model of a single mode energy harvester and properties for optimal power generation," Smart Materials and Structures, vol. 17, p. 065026, November 2008.
[20] J. Liang and W.-H. Liao, "Impedance Modeling and Analysis for Piezoelectric Energy Harvesting Systems," IEEE/ASME Transactions on Mechatronics, vol. 17, no. 6, pp. 1145-1157, December 2012.

[21] M. Ferrari, V. Ferrari, M. Guizzetti, B. Andò, S. Baglio and C. Trigona, "Improved energy harvesting from wideband vibrations by nonlinear piezoelectric converters," Sensors and Actuators A, vol. 162, pp. 425$431,2010$.

[22] D. Zhu, M. J. Tudor and S. P. Beeby, "Strategies for increasing the operating frequency range of vibration energy harvesters: a review," Measurement Science and Technology, vol. 21, p. 022001, 2010.

[23] T. Huguet, M. Lallart and A. Badel, "Bistable vibration energy harvester and SECE circuit: exploring their mutual influence," Nonlinear Dynamics, pp. 485-50197, 2019.

[24] A. Brenes, E. Lefeuvre and C.-S. Yoo, "Experimental validation of wideband piezoelectric energy harvesting based on frequency-tuning synchronized charge extraction," Journal of Physics : Conference Series, vol. 1052, p. 012050, 2018.

[25] C. Richard, D. Guyomar, D. Audigier and H. Bassaler, "Enhanced semi passive damping using continuous switching of a piezoelectric device on an inductor," Smart Structures and Materials : Damping and Isolation, vol. 3989, 2000.

[26] Y. C. Shu and I. C. Lien, "Analysis of power output for piezoelectric energy harvesting systems," Smart Materials and Structures, vol. 15, pp. 1499-1512, 2006

[27] A. Gelb and W. E. V. Velde, Multiple-input describing functions and nonlinear system design, Cambridge: McGraw-Hill Book Company, 1968.

[28] L. Tang and Y. Yang, "Analysis of synchronized charge extraction for piezoelectric energy harvesting," Smart Materials and Structures, vol. 20, p. 085022, 2011.

[29] W. Zhou and L. Zuo, "A Self-Powered Piezoelectric Vibration Control System With Switch Precharged Inductor (SPCI) Method," IEEE/ASME Transactions on Mechatronics, vol. 20, no. 2, pp. 773-781, 2015.

[30] M. Lallart, L. Garbuio, C. Richard and D. Guyomar, "Low-Cost Capacitor Voltage Inverter for Outstanding Performance in Piezoelectric Energy Harvesting," IEEE Transactions on Ultrasonics, Ferroelectrics and Frequency Control, vol. 57, no. 2, pp. 281-291, 2010.

[31] Farnell, March 2017. [Online]. Available: http://fr.farnell.com/inductances.

[32] A. Morel, G. Pillonet, Y. Wanderoild and A. Badel, "Dielectric Losses Considerations for Piezoelectric Energy Harvesting," Journal of Low Power Electronics, vol. 14, pp. 244-254, 2018.

[33] P. Gasnier, J. Willemin, S. Boisseau, G. Despesse and C. Condemine, "An Autonomous Piezoelectric Energy Harvesting IC Based on a Synchronous Multi-Shot Technique," IEEE Journal of Solid-State Circuits, vol. 49, no. 7, pp. 1561-2014, 2014

[34] T. Hehn, F. Hagedorn, D. Maurath, D. Marinkovic, I. Kuehne, A. Frey and Y. Manoli, "A Fully Autonomous Integrated Interface Circuit for Piezoelectric Harvesters," IEEE Journal of Solid-State Circuits, vol. 47, no. 9, pp. 2185 - 2198, 2012.

[35] M. Dini, A. Romani, M. Filippi and M. Tartagni, "A Nano-Power Synchronous Charge Extractor IC for Low Voltage Piezoelectric Energy Harvesting with Residual Charge Inversion," IEEE Transactions on Power Electronics, vol. 31, no. 2, pp. 1263-1274, January 2015. 
[36] TRS Technologies, Inc, “TRS Technologies Resources," December 2018. [Online]. Available: http://www.trstechnologies.com/Resources.

[37] D. Gibus, P. Gasnier, A. Morel, S. Boisseau and A. Badel, "Modelling and design of highly coupled piezoelectric energy harvesters for broadband applications," in PowerMEMS, Daytona Beach, United States, 2018.

[38] W. Q. Liu, A. Badel, F. Formosa and Y. P. Wu, "A new figure of merit for wideband vibration energy harvesters," Smart Materials and Structures, vol. 24, p. 125012, 2015.

[39] S. P. Beeby, R. N. Torah, M. J. Tudor, P. Glynne-Jones, T. O'Donnell, C. R. Saha and S. Roy, "A micro electromagnetic generator for vibration energy harvesting," Journal of Micromechanics and Microengineering, vol. 17, pp. 1257-1265, 2007.

[40] S. Roundy and P. K. Wright, "A piezoelectric vibration based generator for wireless electronics," Smart Materials and Structures, vol. 13, no. 5, p. 1131, 2004.

[41] E. K. Reilly, F. Burghardt, R. Fain and P. Wright, "Powering a wireless sensor node with a vibration-driven piezoelectric energy harvester," Smart Materials and Structures, vol. 20, p. 125006, 2011.

[42] H. Durou, G. A. Ardila-Rodriguez, A. Ramond, X. Dollat, C. Rossi and D. Esteve, "Micromachined bulk PZT piezoelectric vibration energy harvester to improve effectiveness over low amplitude and low frequency vibrations," in PowerMEMS, Leuven, Belgium, 2010.

[43] S. Priya, H.-C. Song, Y. Zhou, R. Varghese, A. Chopra, S.-G. Kim, I. Kanno, L. Wu, D. S. Ha, J. Ryu and R. G. Polcawich, "A Review on Piezoelectric Energy Harvesting: Materials, Methods, and Circuits," Energy Harvesting and Systems, vol. 4, no. 2, 2017.

[44] H. Tanaka, G. Ono, T. Nagano and N. Ohkubo, "Electric Power Generation Using Piezoelectric Resonator for Power-free Sensor Node," in IEEE Custom Integrated Circuits Conference, San Jose, California, 2005.

[45] P. D. Mitcheson, E. M. Yeatman, G. K. Rao, A. S. Holmes and T. C. Green, "Energy Harvesting From Human and Machine Motion for Wireless Electronic Devices," Proceedings of the IEEE, vol. 96, no. 9, p. 1457-1486, 2008.

[46] A. Brenes, E. Lefeuvre, A. Badel, S. Seok and C.-S. Yoo, "Shunt-diode rectifier: a new scheme for efficient low-voltage piezoelectric energy harvesting," Smart Materials and Structures, vol. 28, no. 1, 2018. 